\title{
繁 \\ Ácidos graxos poli-insaturados na dieta de poedeiras: impactos sobre a qualidade dos ovos e saúde humana
}

\author{
[Polyunsaturated fatty acids in the diet of laying hens: effects on egg quality and human health]
}

\section{"Revisão/Review"}

\author{
Verônica Lisboa Santos ${ }^{1 *}$, Tiago Araujo Rodrigues ${ }^{2}$, Marcos Antonio Anciuti ${ }^{3}$, Fernando Rutz ${ }^{2}$
}

${ }^{1}$ Departamento de Zootecnia, Universidade Federal do Paraná, Curitiba-PR, Brasil.

${ }^{2}$ Departamento de Zootecnia, Universidade Federal de Pelotas, Pelotas-RS, Brasil.

${ }^{3}$ Departamento de Zootecnia, Instituto Federal Sul-rio-grandense, Campus Pelotas/Visconde da Graça, Pelotas-RS, Brasil.

*Autor para correspondência/Corresponding author: E-mail: vls_agro@yahoo.com.br

\section{Resumo}

Atualmente já são amplamente documentados os benefícios que a ingestão de ácidos graxos polinsaturados proporcionam à saúde humana, principalmente ácidos graxos da série ômega-3. Entretanto, o baixo consumo de alimentos fontes destes nutrientes, principalmente na população ocidental, tem sido associado à distúrbios cardiovasculares, comprometimento em relação a formação de tecido nervoso, cerebral e ao desenvolvimento da visão. No intuito de aumentar a disponibilidade de alimentos que sejam fontes destes nutrientes à população, diversas pesquisas vêm sendo realizadas visando o enriquecimento de ovos, visto que, são fonte de proteínas, gorduras e micronutrientes que desempenham papel importante na nutrição humana além disso diversos trabalhos tem mostrado que é possível alterar a composição dos ovosa partir da manipulação das dietas das poedeiras. O presente artigo de revisão é proposto visando caracterizar o cenário atual sobre o enriquecimento de ovos com ácidos graxos da série ômega-3, através de diversas fontes de alimentos utilizadas na dieta das aves, bem como seus impactos na nutrição humana.

Palavras-chave: algas; DHA; lipídeos; sensorial.

\begin{abstract}
The benefits that the intake of polyunsaturated fatty acids provide to human health, mainly omega-3 fatty acids, are now widely documented. However, the low consumption of food sources of these nutrients, especially in the Western population, has been associated with cardiovascular disorders and impairment in relation to nervous, brain and eye development. In order to increase the availability of foods that are sources of these nutrients to the population, research has been carried out aiming at egg enrichment, since they are a source of proteins, fats, and micronutrients that play an important role in human nutrition. In addition, several studies have shown that it is possible to change the composition of the eggs from the handling of the diets of laying hens. The present review article proposes to characterize the current scenario on the enrichment of eggs with omega-3 fatty acids through various sources of food used in the diet of birds, as well as their impact on human nutrition.
\end{abstract}

Keywords: algae; DHA; lipids; sensory.

\section{Introdução}

Muitos ensaios realizados em seres humanos e várias experiências com animais demonstram que os ácidos graxos poli-insaturados (AGP's) $\omega-3$ estão envolvidos no desenvolvimento e manutenção de vários órgãos e participam na

prevenção de diferentes patologias. Os três AGP's considerados de maior importância são $o \quad \alpha$ linolénico (18:3 n-3, ALA), o ácido eicosapentaenóico (20:5 n-3, EPA) e ácido docosahexaenóico (22:6 n-3, DHA). O DHA exerce 
importante função no desenvolvimento cerebral e de tecidos da retina em fetos e crianças pequenas, evidenciando a importância de sua adequada ingestão por gestantes (Jordan, 2010). Além disso, os AGP's n-3 têm sido relacionados à prevenção e tratamento de muitas outras doenças crônicas, como doenças neurológicas, câncer, doenças inflamatórias, obesidade e diabetes melittus (Yashodhara et al., 2009).

Ovos são produtos que apresentam alto potencial para enriquecimento com ácidos graxos poli-insaturados, pois, são consumidos pela maioria da população mundial, in natura ou processados. Estudos demonstram que a suplementação da dieta de poedeiras com óleos vegetais de linhaça ou canola, óleo de peixes e, mais recentemente, extratos de algas, aumenta a deposição de ácidos graxos poli-insaturados $\omega$-3 na gema dos ovos $\mathrm{O}$ presente artigo de revisão é proposto visando caracterizar o cenário atual sobre o enriquecimento de ovos com ácidos graxos da série ômega-3, através de diversas fontes de alimentos utilizadas na dieta das aves, bem como seus impactos na nutrição humana.

\section{Ácidos graxos de cadeia longa e a importância para a saúde humana}

A necessidade de produtos alimentares e de ingredientes que proporcionem benefícios, além do seu tradicional valor nutricional, criaram um grande interesse acadêmico e comercial (Yalçin e Ulnal, 2010) por este nicho de mercado. Assim, a prevenção de doenças e a promoção da saúde através do consumo de alimentos funcionais e nutracêuticos têm recebido considerável atenção global, e diferentes produtos, com estas características, já estão disponíveis no mercado.

Todos os essenciais para os seres humanos e devem ser provenientes da dieta (Rubio- Rodriguez et al., 2010). Embora nos animais o 18:2ø-6 e o 18:3 ๑3 possam ser convertidos em AA, EPA e DHA, as taxas de conversão são baixas, portanto são considerados essenciais. É importante ressaltar que as duas séries modulam o metabolismo e o transporte do colesterol, formando parte das lipoproteínas a eles associadas.

O consumo de ácidos graxos poliinsaturados de cadeia longa, em particular os que pertencem à série $₫-3$, EPA e DHA, ocorre principalmente através do consumo de frutos do mar, apresentando níveis geralmente baixos na chamada dieta "ocidental", Schmidt (2001), além disto, estes alimentos também são fontes ricas de outros nutrientes, tais como proteínas, várias vitaminas (por exemplo, A, D, B12) e minerais (por exemplo, iodo e selénio), e outros compostos bioativos, incluindo os carotenóides com propriedades antioxidantes (por exemplo, a astaxantina), fitoesteróis relacionados com os efeitos hipocolesterolémicos, e o aminoácido taurina, ligados a efeitos cardioprotetores (Larsen, 2011).

Os ácidos graxos essenciais de cadeia longa: AA; EPA e DHA fazem parte da estrutura dos fosfolipídeos, que são componentes importantes das membranas e da matriz estrutural de todas as células. Além de seu papel estrutural, esses lipídeos podem também modular a função celular ao atuarem como mediadores intracelulares da transdução de sinais e como moduladores das interações entre células (Carmo e Correia, 2009). Estes três AGPI's foram os mais caracterizados devido aos comprovados efeitos benéficos na saúde humana. O DHA está presente nas membranas cerebrais e na retina; o AA nas membranas cerebrais; e o EPA no sistema cardiovascular (Ward e Singh, 2005).

Entre os efeitos fisiológicos nos humanos do EPA e DHA, estão a prevenção e tratamento de doenças cardiovasculares, hipertensão, inflamações em geral, asma, artrite, psoríase e vários tipos de câncer (Suarez-Mahecha et al., 2002) além de diminuição de arritmias, disfunção endotelial, níveis de triglicerídeos circulantes (Burilo e Mosafarian, 2012).

Carmo e Correia (2009), relatam que alguns mecanismos pelos quais os AGP's $\omega-3$ podem modificar o processo de carcinogênese foram propostos: supressão da biossíntese dos eicosanoides derivados do AA, o que resulta em alteração da resposta imunológica às células tumorais e modulação da inflamação; impacto na proliferação celular, na apoptose, na disseminação de metástases e na angiogênese; influência na atividade do fator de transcrição nuclear, na expressão gênica e nas vias de transdução de sinais, levando a mudanças no metabolismo celular, crescimento e diferenciação das células; alteração no metabolismo do estrogênio, o que gera menor estímulo ao crescimento das células hormônio dependentes; aumento ou diminuição da produção de radicais livres, e relação direta com a sensibilidade à insulina e à fluidez das membranas, embora estes mecanismos careçam de estudos mais aprofundados. Segundo Siddiqui et al. (2007) esses 
lipídeos possuem a capacidade de modificar as funções celulares, modulando a estrutura e função de domínios lipídicos específicos dentro da membrana plasmática.

De acordo com Yalçin e Ulnal (2010), os ácidos graxos $₫-3$ têm um efeito importante na estrutura e função do cérebro, sendo requeridos para diferenciação e funcionamento das células. Deficiências de ALA alteram o desenvolvimento cerebral e perturbam a composição e propriedades físico-químicas das membranas celulares do cérebro. Isto leva a modificações físico-químicas e bioquímicas e induz a perturbações fisiológicas, e resulta em comportamento neurossensorial deprimido. O ALA presente na dieta de recémnascidos (especialmente prematuros) influencia as habilidades visuais, cerebrais, e intelectuais. Além disso, AGPI's $₫-3$ estão envolvidos na prevenção de alguns transtornos neuropsiquiátricos, particularmente depressão, bem como em demência e, em particular, na doença de Alzheimer, em idosos (Bourre, 2004).

Estudos experimentais têm demonstrado que a depleção de DHA no desenvolvimento da retina e do cérebro resulta em diminuição da função visual com anormalidades no eletroretinograma, deficits de aprendizado e alterações no metabolismo de dopamina e serotonina (De $\mathrm{La}$ Presa, 1999).

\section{Fontes}

$\mathrm{O}$ enriquecimento da dieta de poedeiras com óleos e sementes vegetais, óleos e farinhas de peixes e substratos de algas marinhas, proporciona deposição eficiente de ácidos graxos $\infty-3$ nas gemas dos ovos.

\section{Linhaça}

A semente de linhaça (Linum usitatissimum) constitui a espécie vegetal mais rica em $₫-3$, formada praticamente por ALA. O óleo de linhaça é composto por $57 \%$ de ácidos graxos $₫-3,16 \%$ de ๑-6, 18\% de ácidos graxos monoinsaturados e somente $9 \%$ de ácidos graxos saturados. A predominância de $₫$-3 é três vezes superior ao $₫-6$, fazendo com que seja a maior fonte vegetal de ALA, cinco vezes mais abundante que em nozes ou óleo de canola (Ramcharitar et al., 2005; Oomah, 2006).

Desde o final dos anos noventa, ovos enriquecidos com AGP's $₫-3$, produzidos através de suplementação alimentar com linhaça, tornaram-se disponíveis no mercado em muitos países. Dietas enriquecidas com linhaça resultam, principalmente, em aumento no teor de ALA, (até $200 \mathrm{mg}$ por ovo), mas também em um aumento substancial no teor de DHA, (até $90 \mathrm{mg}$ por ovo), com a utilização de $15 \%$ de linhaça (Aymond e Van Elswyk, 1995).

\section{Óleo e farinha de peixes:}

A principal fonte de AGP's $₫$-3 são óleos de peixes gordurosos (Rubio- Rodriguez et al., 2010) pois, se alimentam de fictoplancton e zooplancton (fontes primárias de EPA e DHA). A extração de AGP's dos óleos de peixes é fortemente questionada porque a mesma não conseguirá atender a demanda futura, tem risco de contaminação (com metais pesados, compostos orgânicos e dioxinas), possui sabor e aroma desagradáveis, e apresenta pouca estabilidade oxidativa (Ratledge, 2004). Embora o óleo de peixe contenha EPA, bem como DHA, os ovos de galinhas alimentadas com óleo de peixe são enriquecidos, principalmente, com DHA, enquanto o conteúdo de EPA aumenta em menor proporção (Bovet et al., 2007; Cachaldora et al., 2008; Carrillo et al., 2008). Peixes marinhos geralmente apresentam teores mais elevados de $₫-3$ quando comparados aos peixes de água doce, como pode ser observado na Tabela 1.

Níveis de inclusão acima de 1,5\% de óleo de peixe podem gerar ovos que geralmente apresentam características sensoriais que não são aceitas pelos consumidores ocidentais (GonzalezEsquerra e Leeson, 2000). Contudo, a inclusão dietética de óleo de peixe em níveis em torno de $1,5 \%$ (nível limiar para caracterizar sabores desagradáveis) geralmente resulta em níveis de DHA na gema abaixo de $100 \mathrm{mg} / \mathrm{ovo}$ (Aymond e Van Elswyk, 1995; Gonzalez-Esquerra e Leeson, 2000; Lawlor et al., 2010). Estes níveis não são muito mais elevados do que os obtidos através da suplementação com linhaça. Lipídeos, especialmente ácidos graxos insaturados, têm sido encapsulados visando diminuir a susceptibilidade à oxidação (Favaro-Trindade et al., 2008), ainda assim, a suplementação alimentar da dieta das aves com óleo de peixe microencapsulado, que se esperaria ter maior estabilidade oxidativa, ainda mantém os impactos sensoriais negativos sobre ovos (Lawlor et al., 2010). 
Tabela 1. Conteúdo de AGS $₫$ - 3, especificamente EPA e DHA, em peixes brasileiros de água doce e em peixes marinhos (\% de peso de quantidade total de AGS).

\begin{tabular}{lcc}
\hline \multirow{2}{*}{ Peixe } & \multicolumn{2}{c}{ AG ômega 3 } \\
\cline { 2 - 3 } Água Doce & EPA & DHA \\
\hline Curimba (Prochilodus lineatus) & 5,60 & 3,00 \\
Lambari (Astyanax sp.) & 2,60 & 6,80 \\
Pintado (Pseudoplatystoma corruscans) & 7,50 & 21,80 \\
Traíra (Hoplias spp.) & 3,40 & 7,10 \\
Mandi (Pimelodus maculatus) & 1,50 & 2,00 \\
\hline Marinhos & & \\
\hline Cavalinha (Equisetum spp.) & 6,20 & 13,00 \\
Manjuba (Anchoviella lepidentostole) & 8,80 & 23,70 \\
Pescada (Cynoscion sp.) & 7,70 & 19,20 \\
Arraia (Brycon sp.) & 4,10 & 11,60 \\
Sardinha (Sardinella brasiliensis) & 24,20 & 6,50 \\
Atum (Thunnus sp.) & 7,80 & 32,50 \\
\hline Adaptado de Watannabe (1987).
\end{tabular}

Adaptado de Watannabe (1987).

Algas

As microalgas marinhas têm gerado interesse entre os pesquisadores por serem fonte alternativa de compostos como vitaminas, proteínas com aminoácidos essenciais, polissacárideos, ácidos graxos mono e poliinsaturados, ácidos nucleicos, minerais, e pigmentos fotossintéticos como carotenóides e clorofilas (Raja et al., 2008) e, segundo Ferreira et al. (2013) constituem uma fonte alternativa potencial na obtenção de ácidos graxos essenciais. As microalgas crescem autotroficamente utilizando luz e dióxido de carbono, e também podem ser cultivadas em sistema heterotrófico, usando compostos orgânicos como energia e fonte de carbono, ou ainda em sistema de cultivo mixotrófico (Ferreira et al., 2013). Exemplos incluem Isochrysis galbana e Nannochloropsis oculata, conhecidas por apresentarem alto conteúdo de polissacarídeos solúveis e insolúveis e proteínas, bem como o conteúdo significativo de AGP's. A I. galbana contém grandes quantidades de DHA e a $N$. oculata tem uma percentagem mais elevada de EPA (Brown et al., 1997).

Os AGP's originários das microalgas têm um mercado muito promissor na biotecnologia, em especial na indústria de alimentos funcionais (Bertoldi et al., 2008). De acordo com Fraeye et al. (2012), adição de microalgas como fonte de cadeia AGPI's de cadeia longa à dietas de poedeiras é uma forma eficaz de aumentar o nível de DHA nos ovos. Além disso, esta fonte de suplementação oferece várias vantagens em comparação com óleo de peixe, especialmente no que se refere à estabilidade oxidativa dos lipídeos.

\section{Ovos Enriquecidos}

O ovo é uma complexa entidade química e biológica (Raes et al., 2002). Qureshi et al., (2007) estudaram um grande número de idosos, os quais consumiam um ou mais ovos por dia e não observaram aumento do risco para doença arterial coronariana. Ainda neste sentido, Djoussi e Graziano (2008) indicam não haver correlação entre o aumento do risco de doenças cardiovasculares e acidente vascular cerebral com a ingestão de ovos. Mutunji et al. (2008) demonstraram não haver nenhum efeito sobre as lipoproteínas de baixa densidade (LDL colesterol) com a ingestão diária de dois ovos por seis semanas. De acordo com Ruxton et al. (2010), na medida em que mais pesquisas são realizadas, maior é o estímulo ao consumo de ovos, visto que dados científicos contribuem para esclarecer os efeitos dos alimentos na nutrição humana e no bem-estar das pessoas.

Houwe et al. (2002), adicionaram farinha de atum à dieta de poedeiras e constataram aumento significativo nos valores de DHA nas gemas dos ovos. Após duas semanas recebendo a dieta experimental os níveis de DHA já eram bem superiores, demonstrando rápida incorporação deste ácido graxo às gemas.

Oliveira et al. (2010), avaliaram o perfil lipídico de gemas de ovos de aves alimentadas com três diferentes fontes de lipídeos (óleos de soja, girassol e linhaça) contra um tratamento controle. Os ovos das aves alimentadas com óleo de soja apresentaram maior quantidade de AGPI's da série $\varpi-6$, enquanto os ovos produzidos por aves que consumiram óleo de linhaça apresentaram maior quantidade de AGP's da série $₫-3$. 
Aymond e Van Elswyk (1995) enriqueceram a dieta de poedeiras com $15 \%$ de semente de linhaça moída, constatando que o conteúdo do ácido graxo poli-instaurado ALA dos ovos aumentou de 13 para $212 \mathrm{mg} / \mathrm{ovo}$, enquanto o conteúdo de DHA aumentou de 28 a $90 \mathrm{mg} / \mathrm{ovo}$. Isto indica que as galinhas são capazes de converter ALA em DHA. A maioria dos autores relata que a conversão é bastante limitada, semelhante como em seres humanos. Isto ocorre devido a baixa atividade das enzimas de dessaturação envolvidas na conversão do ALA. Segundo Cachaldora et al. (2008), a eficiência da conversão é afetada por vários fatores, como a presença de quantidades elevadas de AGP's $₫-6$ na dieta (o que aumenta a competição para as enzimas dessaturases, causando uma diminuição na eficiência de conversão do ALA). Fredriksson et al. (2006) relatam que a idade e linhagem das galinhas exercem efeito sobre a eficiência de elongação e dessaturação do ALA. Tem sido postulado que as aves mais velhas possuem o fígado maior, permitindo uma conversão mais eficaz de ALA em DHA. Cachaldora et al. (2008), avaliaram os ovos de galinhas alimentadas com dietas ricas em ambos ALA e EPA/DHA e observaram que tanto o exceso quanto a deficiência de ácidos graxos de cadeia longa limitam a conversão de ALA, concluindo que a eficiência de conversão depende principalmente da quantidade total de AGP's $₫-3$ na dieta, bem como suas proporções relativas.

Saphira et al. (2008) ao contrastarem o teor lipídico de ovos de aves alimentadas com a inclusão de $5 \%$ de linhaça contra um grupo controle, observaram que os ovos do grupo suplementado com linhaça resultaram em um aumento de 3,8 vezes no total de AGP's $₫-3,6,4$ vezes de ALA, e 2,4 vezes de DHA após cinco semanas de experimento.

Bruneel et al. (2013), suplementaram a dieta de poedeiras com microalgas (tratamento controle, $5 \%$ e $10 \%$ de inclusão de microalgas) e analisaram o teor lipídico dos ovos nos dias 0 (zero), 14 e 28 dias (término da suplementação) e 42 dias após o início do experimento. Os autores observaram que houve aumento no teor de ácidos graxos $₫-3$ nos ovos das aves alimentas com as microalgas nos períodos de 14 e 28 dias, diferindo do tratamento controle, porém, não houve diferença estatística entre os teores de ácidos graxos poli-insaturados, quando comparados os resultados de 14 e 28 dias. A análise realizada aos 42 dias, quando todas as aves já estavam sendo alimentadas com a dieta controle há 14 dias, demonstrou que o nível de ácidos graxos poli-insaturados diminuiu e foi compatível ao do tratamento controle para todos os grupos avaliados.

Lemahieu et al. (2015), avaliaram o perfil lipídico de ovos de poedeiras Isa Brown, testando quatro fontes de ácidos graxos poli-insaturados ômega-3 (linhaça, Isochrysis galbana, óleo de peixe e DHA ouro ${ }^{\circledR}$ ) de tal maneira que a mesma quantidade extra de AGPI $₫-3$ (120 mg por $100 \mathrm{~g}$ de alimentação) foi adicionado à dieta. A menor eficiência de enriquecimento (aproximadamente $6 \%$ ) foi observada quando a linhaça (fonte de ALA) foi adicionada. Níveis drasticamente superiores de enriquecimento foram observados com a suplementação das outras três fontes de AGP's, diferindo entre si no teor de enriquecimento, sendo aproximadamente 55\% para o óleo de peixe, $30 \%$ para Isochysis galbana e $45 \%$ para o DHA ouro ${ }^{\circledR}$, o que os autores atribuiram às diferentes bio-acessibilidades dos perfis de AGPI $₫-3$ das três fontes.

Fredriksson et al. (2006), adicionaram Nannochloropsis oculata, uma espécie de microalga contendo tanto EPA e ALA ,à alimentação das galinhas, observaram baixas concentrações de EPA e aumento significativo no nível de DHA na gema dos ovos, especialmente na fração fosfolipídica, em detrimento dos ácidos graxos poli-insaturado da série $₫-6$.

Lawlor et al. (2010) avaliaram a transferência de DHA e EPA e atributos sensoriais de ovos produzidos por aves recebendo quatro dietas, com diferentes níveis de inclusão de óleo de peixe microencapsulado (OPM), nas doses de 0 (controle), 20, 40, ou $60 \mathrm{~g} / \mathrm{kg}$ de ração do produto. As dietas foram oferecidas durante 21 dias e os autores observaram aumento de $141 \mathrm{mg} / \mathrm{gema}(0$ $\mathrm{g} / \mathrm{kg}$ de OPM) para $299 \mathrm{mg} / \mathrm{gema}(60 \mathrm{~g} / \mathrm{kg}$ OPM). As diferenças de atributos sensoriais entre os tratamentos pareceram ser dependentes do método de preparação (cozidos ou mexidos). Diferenças significativas entre os tratamentos foram encontrados para amostras cozidas, as quais foram classificadas com maior percepção de sabores desagradáveis e "sabor de enxofre". Nenhuma diferença sensorial significativa foi encontrada nas amostras de ovos mexidos. Os resultados fornecem evidências de que o uso de óleo de peixe microencapsulado pode servir como um veículo eficaz para aumento do teor de AGPI's da série $₫$ 3 , no entanto, o potencial para alguns atributos 
sensoriais adversos está presente nos níveis mais elevados de incorporação.

Gonzales-Esquera e Leeson (2000), relatam que inclusão de até $60 \mathrm{~g} / \mathrm{kg}$ óleo desavelha (Alosa fallax) (contendo $110 \mathrm{~g} / \mathrm{kg}$ EPA e $90 \mathrm{~g} / \mathrm{kg}$ DHA) em rações de poedeiras produziram ovos contendo aproximadamente 150-200 mg de DHA/ovo e 45$60 \mathrm{mg}$ de EPA/ovo.

\section{Razão entre os AGP,' ômega-6 e ômega-3 e Recomendações de Ingestão Diária (RID)}

A razão entre AGP's $₫-6$ e $₫-3$ é importante para a saúde humana, uma vez que há competição entre as enzimas envolvidas no alongamento e dessaturação, tanto do ácido linoléico quanto do ácido alfa-linolênico, os quais não podem se interconverter (Brandão et al. 2005; Simopoulos, 2006).

Segundo Ramin et al. (2013), atualmente os índices de relação entre $\omega-6 / \infty-3$ nas dietas ocidentais pode chegar de 10:1 à 25:1, resultado, provavelmente, do grande consumo de produtos industrializados e fast foods, podendo estar relacionado ao elevado índice de obesidade observado na população ocidental. Uma proporção de 4:1, ou menos é considerada ótima no alongamento de cadeia de $11 \mathrm{~g}$ de ácido alfalinolênico em $1 \mathrm{~g}$ de EPA. O Instituto do Coração de Lyon (França), recomenda a razão entre $₫-6: Ф-$ 3 de 4:1 (Simopoulos, 2000; Simopoulos, 2006). Em ovos enriquecidos com óleo de peixe e óleo de canola é possível encontrar proporção ainda menor, podendo chegar a 2:1 (Lewis et al., 2000; Mazalli et al., 2004).

De acordo com Bertechini e Mazzuco (2015), a relação ๑-6/@-3 são de 7:1 em ovos regulares, nos ovos enriquecidos com AGP's $₫-3$, os valores variam ao redor de 3,7:1, ou menos. Portanto, atende a recomendação dietética (Simoupoulos, 2008; Deckelbaum, 2010).

Atualmente, as agências internacionais divergem a respeito da recomendação de ingestão diária, como pode ser observado na Tabela 2.

Tabela 2. Recomendação de ingestão diária dos AGPI $₫ 6$ e $\oplus 3$ segundo diferentes agências internacionais.

\begin{tabular}{|c|c|c|c|c|c|}
\hline & EFSA $^{1}$ & ISSFAL $^{2}$ & $\mathrm{AHA}^{3}$ & $\mathrm{USDA}_{\mathrm{e}} \mathrm{HHS}^{4}$ & $\mathrm{AHN}^{5}$ \\
\hline Adultos saudáveis & $\begin{array}{l}250 \mathrm{mg} / \mathrm{dia} \\
\text { EPA/DHA }\end{array}$ & & $\begin{array}{l}\text { 500mg/dia } \\
\text { EPA/DHA }\end{array}$ & & 250mg/dia EPA/DHA \\
\hline $\begin{array}{l}\text { Grávidas e } \\
\text { Lactantes }\end{array}$ & $\begin{array}{l}\text { 250mg EPA } \\
+ \text { DHA/dia, } \\
+100-200 \mathrm{mg} \\
\text { DHA }\end{array}$ & & & $\begin{array}{l}\text { Consumir entre } 12 \\
\text { porções de frutos } \\
\text { do mar por } \\
\text { semana, (baixos } \\
\text { em metil- } \\
\text { mercúrio) }\end{array}$ & $\begin{array}{c}300 \mathrm{mg} / \text { dia de EPA/ } \\
\text { DHA, dos quais, pelo } \\
\text { menos, 200mg/dia } \\
\text { deverão ser DHA }\end{array}$ \\
\hline
\end{tabular}

\begin{tabular}{|c|c|c|c|}
\hline $\begin{array}{l}\text { Adultos com } \\
\text { doença } \\
\text { cardiovascular }\end{array}$ & $\begin{array}{c}\text { 500mg/ } \\
\text { dia } \\
\text { EPA/DHA }\end{array}$ & & \\
\hline $\begin{array}{l}\text { População em } \\
\text { geral }\end{array}$ & & $\begin{array}{l}\text { Duas poções } \\
\text { de peixe } \\
\text { "gordo" por } \\
\text { semana }\end{array}$ & $\begin{array}{c}250 \text { mg/dia EPA } \\
\text { e DHA }\end{array}$ \\
\hline
\end{tabular}

\begin{tabular}{lc}
\hline $\begin{array}{l}\text { Pessoas com } \\
\text { doenças } \\
\text { coronarianas }\end{array}$ & $\begin{array}{c}1000 \mathrm{mg} / \mathrm{dia} \\
0-3 \mathrm{EPA} / \mathrm{DHA}\end{array}$ \\
\hline Pessoas com & $2000 \mathrm{a} 4000$ \\
triglicerídeos & $\mathrm{mg} / \mathrm{dia} \omega-3$ \\
elevados & EPA/DHA \\
\hline 'European Food Safety Authority, 2010; ${ }^{2}$ ISSFAL (International Society for the Study of Fatty Acids and Lipids) 2004; ${ }^{3} \mathrm{AHA}$ \\
American Heart Association, 2002; ${ }^{4}$ USDA US Department of Agriculture and HHS Department of Health and Human Services; \\
${ }^{5}$ Acids in Human Nutrition 2010. Fonte: adaptado de Lichestein et al. (2006); Interim Summary of Conclusions and Dietary \\
Recommendations on Total Fat \& Fatty Acids; Scientific Opinion on Dietary Reference Values for fats, including saturated fatty \\
acids, polyunsaturated fatty acids, monounsaturated fatty acids, trans fatty acids, and cholesterol.
\end{tabular}

Como esse desequilíbrio é promotor de doenças graves, diferentes organizações internacionais recomendaram a introdução de ARA ou AA, EPA e DHA nas fórmulas infantis e nos suplementos nutricionais para adultos e para mulheres grávidas ou em aleitamento.

Estudos de prevenção secundária, utilizando $850 \mathrm{mg}$ a 4 g EPA e DHA diariamente têm 
demonstrado uma redução significativa da mortalidade total e morte súbita (20\% a $50 \%$ ) com tratamentos de duração de 12 a 42 meses, em pacientes com doença coronariana ou infarto do miocárdio prévio (Jacobsen, 2006). Deve notar-se que os ensaios clínicos de intervenção que envolvem consumo de AGPi' $₫$-3 durante períodos limitados mostram benefícios cardiovasculares limitados quando comparados com os resultados de estudos epidemiológicos com populações caracterizadas por um tempo de vida que consomem dietas ricas em nutrientes (Robinson, 2006). Além disso, a variação dos efeitos da suplementação na prevenção primária e secundária da doença coronariana entre os estudos é afetada por vários fatores, incluindo a dieta basal, meio de suplementação, os fatores genéticos e etnia.

\section{Ovos enriquecidos com $0-3$ e a Legislação}

De acordo com Kus e Mancini-Filho (2010), há um crescente apelo nas embalagens de alimentos pela utilização de "anúncios" sobre os benefícios dos ácidos graxos poli-insaturados de cadeia longa (AGPI-CL). A O Regulamento da Comissão Europeia 116/2010, relativao à reinvindicações sobre o uso de propagandas em alimentos relacionadas à nutrição e a saúde, estabelece que para rotular um produto como "Elevado teor em AGP's œ-3", o mesmo deve conter cerca de $6 \mathrm{~g}$ de ácido linoleico ALA por $\mathrm{kg}$ de alimento ou $100 \mathrm{kcal}$ ou $0,80 \mathrm{~g}$ de EPA + DHA, em $1 \mathrm{~kg}$ ou $100 \mathrm{kcal}$ de alimentos (Lamas et al., 2015).

A ANVISA (2008) estabelece que: "O consumo de ácidos graxos $\omega-3$ auxilia na manutenção de níveis saudáveis de triglicerídeos, desde que associado a uma alimentação equilibrada e hábitos de vida saudáveis",

De acordo com a Resolução da Diretoria Colegiada $\mathrm{N}^{\circ}$ 54, de 12 de novembro de 2012, para que um alimento possa trazer em seu rótulo a alegação "fonte de ômega 3" deve conter o mínimo de $300 \mathrm{mg}$ de ácido alfa-linolênico por $100 \mathrm{~g}$ ou $100 \mathrm{ml}$ em pratos preparados conforme o caso ou o mínimo de $40 \mathrm{mg}$ da soma de EPA e DHA por porção. Para que a alegação "alto conteúdo de ômega 3" seja válida, o alimento deve conter o mínimo de $600 \mathrm{mg}$ de ácido alfa-linolênico por 100 $\mathrm{g}$ ou $100 \mathrm{ml}$ em pratos preparados conforme o caso ou o mínimo de $80 \mathrm{mg}$ da soma de EPA e DHA por porção (Brasil, 2012).

\section{Considerações finais}

Os efeitos benéficos provenientes da ingestão de AGPI's $₫$-3 à saúde podem ser atribuídos principalmente ao EPA e DHA. Óleos ou farinhas de peixe são ricas fontes de DHA, contudo, diversas pesquisas demonstram que a suplementação de dietas de aves com estes produtos leva à rejeição dos ovos pelos consumidores devido à transferência de sabor e odor desagradáveis ao paladar humano. Pesquisas mais recentemente demonstram que microalgas marinhas constituem-se em uma fonte eficaz para aumentar o nível de DHA em ovos, oferecendo vantagens em relação ao óleo de peixe como a não transferência de sabor ou odor aos ovos e maior estabilidade oxidativa dos lípideos, contudo, são necessários estudos mais aprofundados que abranjam os diferentes gêneros e espécies de microalgas, bem como sua composição e seus impactos sobre o metabolismo das aves e qualidade dos ovos. O enriquecimento de ovos com AGPI's da série $₫-3$ constitui-se em uma alternativa importante para incrementar a ingestão destes nutrientes, tornando-os acessíveis à dieta de grande parte da população.

\section{Referências}

ANVISA - Agência Nacional de Vigilância Sanitária. Alimentos com alegações de propriedades funcionais e ou de saúde, novos alimentos/ingredientes, substâncias bioativas e probióticos. Lista de alegações de propriedade funcional aprovadas. 2008. Disponível em: <http://portal.anvisa.gov.br/alimentos/alegac oes>. Acesso em: 25 nov. 2017.

Aymond W.M.; Van Elswyk, M.E. Yolk thiobarbituric acid reactive substances and n3 fatty acids in response to whole and ground flaxseed. Poultry Science, (74): 1388-1394, 1995.

Bertechini, A.G.; Mazzuco, H. Ovo de consumo: uma revisão. Avicultura Industrial, 2: 40-47, 2015.

Bertoldi, F.C.; Sant'Anna, E. e Oliveira, J.L.B. Revisão: Biotecnologia de microalgas. Boletim do Centro de Pesquisa e Processamento de Alimentos, 26(1): 9-20, 2008.

Borges, M.C.; Santos, F.M.M.; Telles, R.W.; Correia, M.I.T.D.; Lanna, C.C.D. Ácidos graxos poli-insaturados ômega-3 e lúpus eritematoso sistêmico: o que sabemos? 
Revista Brasileira de Reumatologia, 54(6): 459-466, 2014.

Bourre, J.M. Roles of unsaturated fatty acids (especially omega-3 fatty acids) in the brain at various ages and during ageing. Journal of Nutrition and Health, 8(3): 163-174. 2004.

Bovet, P.; Faeh, D.; Madeleine, G.; Viswanathan, B.; Paccaud, F. Decrease in blood triglycerides associated with the consumption of eggs of hens fed with food supplemented with fish oil. Nutrition, Metabolism \& Cardiovascular Diseases, 17(4): 280-287, 2007.

Brandão, P.A.; Costa, F.G.P.; Barros, L.R.; Nascimento, J.A.J. Ácidos graxos e colesterol na alimentação humana. Agropecuária Técnica, 26(1): 5-14, 2005.

BRASIL. Agência Nacional de Vigilância Sanitária. Resolução da Diretoria Colegiada, RDC $\mathbf{N}^{\circ}$ 54, de 12 de novembro de 2012. Disponível em: <http://portal.anvisa.gov.br/documents/\%203 3880/2568070/rdc0054_12_11_2012.pdf/c5a c23fd-974e-4f2c-9fbc-48f7e0a31864>.

Acesso em 12 jun. 2018.

Brown, M.R., Jeffrey, S.W. Volkman, J.K., Dunstan, G.A. Nutritional properties of microalgae for mariculture. Aquaculture, 151(1): 315-331, 1997.

Bruneel, C., Lemahieu, C., Fraeye, I., Ryckebosch, E., Buyse, J., Muylaert, K., Foubert, I. Impact of microalgal feed supplementation on omega-3 fatty acid enrichment of hen eggs. Journal of Functional Foods, 5(2): 897-904, 2013.

Burillo, E.; Martin-Fuentes, P.; Mateo-Gallego, R.; Baila-Rueda, L.; Cenarro, A.; Ros, E.; Civieira, F. Omega-3 fatty acids and HDL. How do they work in the prevention of cardiovascular disease? Current Vascular Pharmacology, 10(4): 432-441, 2012.

Cachaldora, P.; Garia-Rebollar, P.; Alvarez, C.; Mendez, J.; \& De Blas, J.C. Double enrichment of chicken eggs with conjugated linoleic acid and n-3 fatty acids through dietary fat supplementation. Animal Feed Science and Technology, 144(3): 315-326, 2008.

Carrillo, S.; Lopez, E.; Casas, M.M.; Avila, E.; Castillo, R.M.; Carranco, M.E.; Calvo, C.; Pérez-Gil, F. Potential use of seaweeds in the laying hen ration to improve the quality of $n-3$ fatty acid enriched eggs. Journal of Applied Phycology, 20: 721-728, 2008.

Deckelbaum, R.J. n-6 and n-3 Fatty acids and atherosclerosis: ratios or amounts? Arteriosclerosis, Thrombosis, and Vascular Biology, 30(12): 2325-23266, 2010.

De La Presa, O.S.; Innis, S.M. Docosahexaenoic and arachidonic acid prevent a decrease in dopaminergic and serotoninergic neurotransmitters in frontal cortex caused by a linoleic and alpha-linolenic acid deficient diet in formula-fed piglets. The Journal of Nutrition, 129(11): 2088-2093, 1999.

Djousse, L.; Graziano, J.M. Egg consumption and risk of heart failure in the Physicians Health Study. Circulation, 117(4): 512-516, 2008.

FAO/WHO. Food and Agriculture Organization of the United Nations/World Health Organization. Interim Summary of Conclusions and Dietary Recommendations on Total Fat \& Fatty Acids; From the Joint FAO/WHO Expert Consultation eon Fats and Fatty Acids in Human Nutrition, November 10-14, 2008, WHO HQ, Geneva. Disponível em: $<$ http://www.fao.org/ag/agn/nutrition/docs/Fa ts\%20and\%20Fatty\%20Acids\%20Summary. pdf>. Acesso em: 20 abr. 2017.

Favaro-Trindade, C.S.; Pinho, S.C.; Rocha, G.A. Revisão: Microencapsulação de ingredientes alimentícios. Brazilian Journal of Food Technology, 11(2): 103-112, 2008.

Ferreira, S.P.; Souza-Soares, L.; Costa, J.A.V. Revisão: microalgas: uma fonte alternativa na obtenção de ácidos gordos essenciais. Revista Brasileira de Ciência Animal, 36(3): 275287, 2013

Fraeye, I.; Brunell, C.; Lemahieu, C.; Buyse, J.; Muylaert, K.; Fouber, I. Dietary enrichment of eggs with ômega-3 fatty acids: A review. Food Research International, 48: 961-969, 2012.

Fredriksson, S.; Elwinger, K.; Pickova, J. Fatty acid and carotenoid composition of egg yolk as an effect of microalgae addition to feed formula for laying hens. Food Chemistry, 99(3): 530-537, 2006.

Gonzales-Esquera, R.; Leeson, S. Effect of feeding hens regular or deodorized menhaden oil on production parameters, yolk fatty acid profile and sensory quality of eggs. Poultry Science, 79(11): 1597-1602, 2000. 
Howe, P.R.C.; Downing, J.A.; Grenyer, B.F.; Bryden, W.I. Tuna fishmeal as a source of DHA for n-3 PUFA enrichment of pork, chicken, and eggs. Lipids, 37:1067-1076, 2002.

ITALIA. EFSA. European Food Safety Authority Panel on Dietetic Products, Nutrition, and Allergies (NDA); Scientific Opinion on Dietary Reference Values for fats, including saturated fatty acids, polyunsaturated fatty acids, monounsaturated fatty acids, trans fatty acids, and cholesterol. European Food Safety Authorithy Journal 2010; 8(3):1461. [107 pp.].

Jacobson, T.A. Secondary prevention of coronary artery disease with omega-3 fatty acids. American Journal of Cardiology, 98(4): 6170, 2006.

Jordan, R.G. Prenatal omega-3 fatty acids: Review and recommendations. Journal of Midwifery Women's Health, 55(6): 520-528, 2010.

JOURNAL COMMISSION REGULATION (EU) No 116/2010 of 9 February 2010. Amending Regulation (EC) No 1924/2006 of the European Parliament and of the Council with regard to the list of nutrition claims. Official of the European Union, L37, 16-18. $2010 . \quad$ Disponível em: $<$ https://www.fsai.ie/uploadedFiles/Reg116_ 2010.pdf>. Acesso: em jan. 2018.

Komprda, T. Eicosapentaenoic and docosahexaenoic acids as inflammationmodulating and lipid homeostasis influencing nutraceuticals: a review. Journal of Functional Foods, 4: 25-38, 2012.

Kus, M.M.M.; Mancini-Filho. Funções plenamente reconhecidas de nutrientes - ácidos graxos: eicosapentaenoico (EPA) e docosahexaenoico (DHA). Série de Publicações International Life Sciences Institute Brasil, 17: 3-19, 2010.

Lamas, A.; Antom, X.; Miranda, J.M.; RocaSaavedra, P.A.; Cardelle-Cobas, A.; Rodriguez, J.A.; Franco, C.M.; Cepeda, A. Technological development of functional egg products by an addition of n-3 polyunsaturated-fatty-acid-enriched oil. CyTA- Journal of Food, 14(2): 289-285, 2016.

Larsen, R.; EIlertsen, K.E.; Elvevoll, E.O. Health benefits of marine foods and ingredients. Biotechnology Advances, 9: 508-518, 2011.
Lawlor, J.B.; Gaudett, E.N.; Dickson, T.; House, J.D. Fatty acids profile and sensory characteristics of table eggs from laying hens fed diets containing microencapsulated fish oil. Animal Feed Science and Technology, 156(3): 97-103, 2010.

Lemahieu, C.; Bruneel, C.; E. Ryckebosch, K. Muylaert, J. Buyse, and I. Foubert. Impact of different omega-3 polyunsaturated fatty acid (n-3 PUFA) sources (flaxseed, Isochrysis galbana, fish oil and DHA Gold) on n-3 LCPUFA enrichment (efficiency) in the egg yolk. Journal of Functional Foods, 19: 82818287, 2015.

Lewis, N.M.; Seburg, S.; Flanagan, N.L. Enriched egg as a source of n-3 polyunsaturated fatty acids for humans. Poultry Science, 79(7): 971-974, 2000.

Lichtenstein, A.H.; Appel, L.J.; Brands, M.; Carnethon, M.; Daniels, S.; Franch, H.A. Diet and lifestyle recommendations revision - A scientific statement from the American Heart Association Nutrition Committee. Circulation, 114(1): 82-96, 2006.

Mazalli, M.R.; Faria, D.E.; Salvador, D. A comparison of the feeding value of different sources of fats for laying hens: 1 . Performance characteristics. The Journal of Applied Poultry Research, 13(2): 274-279, 2004.

Mutungi, G.; Ratliff, J.; Puglisi, M.; TorresGonzalez, M.; Vaishnav, U.; Leite, J.O.; Quann, E.; Volek, J.S.; Fernandez, M.L. Dietary cholesterol from eggs increases plasma HDL cholesterol in overweight men consuming a carbohydrate-restricted diet. The Journal of Nutrition, 138(2): 272-276, 2008.

Oliveira, D.D.; Baião, N.C.; Cancado, V.S.; Grimaldi, R.; Souza, M.R.; Lara, L.J.C.; Lana, A.M.Q. Effects of lipid sources in the diet of laying hens on the fatty acid profiles of egg yolks. Poultry Science, 89(11): 2484-2490, 2010.

Oomah, B.D.; Der, T.J.; Godfrey, D.V. Thermal characteristics of Flaxseed (Linum usitatissimum L.) proteins. Food Chemstry, 98(4): 733-741, 2006.

Qureshi, A.I.; Suri, M.F.K.; Ahmed, S.; Nasar, A.; Divani, A.A.; Kirmani, J.F. Regular egg consumption does not increase the risk of stroke or cardiovascular disease. Medical Science Monitor, 13(1): 1-8, 2007.

Raes, K.; Huyghebaert, G.; De Smet, S.; Nollet L.; Arnouts, S.; Demeyer, D. The Deposition of 
Conjugated Linoleic Acids in Eggs of Laying Hens Fed Diets Varying in Fat Level and Fatty Acid Profile. Journal of Nutrition, 132(2): 182-189, 2002.

Raja, R.; Hemaiswarya, S.; Kumar, N.A.; Sridhar, S.; Rengasamy, R.A. Perspective on the biotechnological potential of microalgae. Critical Reviews in Microbiology, 34(2): 77-88, 2008.

Ramcharitar, A.; Badrie, N.; Mattfeldt-Beman, M.; Matsuo, H.; Ridley, C. Consumer acceptability of with muffind flaxseed (Linum usitatissimum). Journal of Food Science, 70(7): 504-507, 2005.

Ramin, I.; Roya, K.; Sharareh, B.; Kianoush, K.D.; Sanam, F. Comparison of long chain polyunsaturated fatty acid content in human milk in preterm and term deliveries and its correlation with mothers' diet. Journal of Research in Medical Science, 18(1): 1-5, 2013.

Ratledge, C. Fatty acid biosynthesis in microorganisms being used for single cell oil production. Biochimie Journal, 86(11): 807815, 2004.

Robinson J.G.; Stone, N.J. Antiatherosclerotic and antithrombotic effects of omega-3 fatty acids. American Journal of Cardiology, 98(4): 3949, 2006.

Rubio-Rodríguez, N.; Beltrán, S.; Jaime, I., De Diego, S., Sanz, M.T., Rovira, J. Production of omega-3 polyunsaturated fatty acid concentrates: a review. Innovative Food Science and Emerging Technologies, 11(1): 1-12, 2010.

Ruxton, C.H.S.; Derbyshire, E.; Gibson, S. The nutritional properties and health benefits of eggs. Food, Science \& Nutrition, 40(3): 263 279, 2010.

Saphira, N.; Weill, P.; Loewenbach, R.A. Egg fortification with $\mathrm{n}-3$ polyunsaturated fatty acids (PUFA): Nutritional benefits versus High n-6 PUFA western diets, and consumer acceptance. The Israel Medical Association Journal, 10(4): 262-265, 2008.

Schmidt, E.B.; Christensen, J.H.; Aardestrup, I.; Madsen, T.; Riahi, S.; Hansen, V.E.; Skou,
H.A. Marine $n-3$ fatty acids: Basic features and background. Lipids, 6: 65-68, 2008.

Siddiqui, R.A.; Harvey, K.A.; Zaloga, G.P. Modulation of lipid rafts by omega-3 fatty acids in inflamation and cancer: implications for use of lipids during nutricional support. Nutrition in Clinical Practice, 22(1): 74-88, 2007.

Simopoulos, A.P. Symposium: role of poultry products in enriching the human diet with n-3 PUFA. Poultry Science, 79(7): 961-970, 2000.

Simopoulos, A.P. The omega-6/omega-3 ratio: the scientific evidence and the need to return the omega-3 fatty acids into eggs and other foods. In: Sim, J.; Sinwoo, H.H. (ed). The amazing egg: nature's perfect functional food for health promotion. Edmonton: University of Alberta, p.195-218, 2006.

Simopoulus, A.P. The omega-6/omega-3 fatty acid ratio, genetic variation, and cardiovascular disease. Asia Pacific Journal of Clinical Nutrition, 17(1): 131-4, 2008.

Souza Carmo, M.C.N.; Correia, M.I.D.T.A. Importância dos Ácidos Graxos Ômega-3 no Câncer. Revista Brasileira de Cancerologia, 55(3): 279-28, 2009.

Suarez-Mahecha, H.; Francisco, A.; Beirão, L.H.; Block, J.M.; Sacco, L.A.; Pardo-Carrasco, S. A importância dos ácidos graxos poliinsaturados presentes em peixes de cultivo e de ambiente natural para a nutrição humana. Boletim do Instituto de Pesca, 28(1): 101110 ,

Ward, O.P.; Singh, A. Omega-3/6 fatty acids: alternative sources of production. Process Biochemistry, 40(12): 3627-3652, 2005.

Yalçin, H.; Unal, M.K. The enrichment of hen eggs with ômega-3 fatty acids. Journal of Medicinal Food, 13(3): 610-614, 2010.

Yashodhara, B.M.; Umakanth, S.; Pappachan, J.M.; Bhat, S.K.; Kamath, R.; Choo, B.H. Omega-3 fatty acids: A comprehensive review of their role in health and disease. Postgraduate Medical Journal, 85(1000): 84-90, 2009. 